\title{
Indicaciones para el uso de terapia de reemplazo hormonal oral en mujeres menopáusicas mayores de 50 años
}

\author{
Donald Fernández Morales
}

\section{Resumen}

El uso de la terapia de reemplazo hormonal oral ha sido una controversia en los últimos años, como consecuencia de una serie de estudios aleatorios que contradicen los lineamientos anteriores. Algunos autores refieren sus observaciones respecto a estas investigaciones, así como las limitaciones. Sin embargo, se demuestra que las únicas indicaciones que persisten para su uso son el tratamiento del síndrome menopáusico, al menor tiempo y la menor dosis posible, y como segunda elección, la osteoporosis. El objetivo de esta revisión es ofrecer un apoyo a los profesionales en medicina que prescriben terapia de reemplazo hormonal en mujeres menopáusicas mayores de 50 años.

Descriptores: Terapia de reemplazo hormonal, síntomas vasomotores, atrofia urogenital, tromboembolismo, cáncer de seno invasivo, enfermedad cerebrovascular

Key words: hormone replacement therapy; vasomotor symptoms; urogenital atrophy; thromboembolism; invasive breast cancer.

Recibido: 6 de septiembre de 2006

Aceptado: 17 de octubre de 2006

Servicio de ginecología y obstetricia. Hospital México, Caja Costarricense de Seguro Social.

Abreviaturas: HERS, estudio de corazón y reemplazo de estrógenos-progestinas; PEPI, ensayo sobre la intervención de estrógenos y progesterona en postmenopausia; OR, razón de ocurrencia; $\mathrm{WHI}$, iniciativa de salud de las mujeres; RR, riesgo relativo

Correspondencia: Donald Fernández Morales. Apartado postal: 1077-I0II Y Griega, San José. Correo electrónico: donaldf@costarricense.cr

ISSN 0001-6002/2007/49/1/24-30 Acta Médica Costarricense, $\odot 2007$ Colegio de Médicos y Cirujanos
En 1932 se reconoce el uso de estrógenos sin oposición de progesterona como tratamiento de los síntomas menopáusicos, aunque su prescripción data desde $1960 .{ }^{1}$ En 1975 Ziel y Finkle reportan el aumento de carcinoma de endometrio en usuarias de estrógenos conjugados, que se incrementa con la duración de la exposición², lo que motiva desde 1981 el uso de progestágenos como protección endometrial. Hace 17 años se inicia el tratamiento continuo de estrógenos y progesterona, pues el cumplimiento hacia la terapia hormonal oral cíclica no era el adecuado. Las principales causas de abandono eran: sangrado uterino irregular, mastalgia, edema, ganancia de peso, irritabilidad, depresión y razones personales. ${ }^{3}$ Esta terapia produce amenorrea en el 56$75 \%$ de las pacientes a 6 meses con endometrios atróficos por histología ${ }^{4,5}$, y alivio de los bochornos en más del 70\%. No está claro si la terapia continua es superior a la cíclica en el mejoramiento de los síntomas vasomotores. ${ }^{3,6}$ Pero Eiken y Kolthoff mencionan que el $87 \%$ de las pacientes con terapia de reemplazo hormonal continua persiste en su cumplimiento a 10 años. ${ }^{7}$

Desde 1992 varias asociaciones médicas señalan lineamientos que prescriben terapia de reemplazo hormonal para mujeres con riesgo de enfermedad cardiaca y osteoporosis, con base en estudios observacionales y experiencia clínica ${ }^{8-11}$ Las investigaciones mostraban que las mujeres que tomaban estrógenos o estrógenos y progesterona, tenían una disminución del riesgo de enfermedad cardiaca de un 35\% a un 50\%. ${ }^{12}$ Grimes y Lobo refieren que estas pacientes muestran el sesgo de la selección: mejor nutrición, más ejercicio, menor fumado y mejor perfil de salud. Además, se menciona el sesgo de adherencia, pues las usuarias continuaban su 
medicamento a pesar de los efectos adversos, con lo que mejoraba su salud, a diferencia de la de quienes ignoraban las prescripciones médicas. El sesgo de seguimiento puede desempeñar su papel en los resultados, pues esta población de pacientes consulta con sus médicos con mayor frecuencia que quienes no toman tratamiento hormonal. También si desarrollan alguna enfermedad intercurrente, es sugestivo que suspendan la terapia hormonal por iniciativa propia o indicación médica (sesgo de sobrevivencia). ${ }^{13}$ Todas estas consideraciones podrían explicar los beneficios cardiovasculares encontrados en los estudios observacionales.

El 37.6\% de las mujeres estadounidenses entre los $50 \mathrm{y}$ los 74 años, sin historia personal de cáncer de seno, usaban terapia de reemplazo hormonal, práctica que era más frecuente en pacientes con histerectomía previa. $^{14}$ Sin embargo, el grado de cumplimiento hacia la terapia era bajo. Ravnikar menciona que el 20\% de 2500 mujeres de 45 a 55 años cesó el tratamiento de terapia de reemplazo hormonal oral a los 9 meses, el 10\% lo usó en forma intermitente y el 20-30\% ni siquiera llenó la receta; la principal razón fue el temor al cáncer. ${ }^{15}$

Se ha señalado el uso de estrógenos y progesterona oral como tratamiento de reemplazo hormonal, para las siguientes condiciones: 1-tratamiento de síntomas vasomotores asociados con la menopausia; 2-prevención de eventos cardiovasculares; 3- tratamiento de la atrofia vaginal y vulvar; 4- prevención de la osteoporosis posmenopáusica.

El objetivo de esta revisión es comentar la evidencia encontrada en la bibliografía médica sobre las indicaciones de los estrógenos conjugados y el acetato de medroxiprogesterona oral, a la dosis más frecuente, en mujeres menopáusicas mayores de 50 años, con el propósito de que sirva como guía a todos los profesionales en medicina que prescriben terapia hormonal oral.

\section{Tratamiento de síntomas vasomotores asociados con la menopausia}

El síntoma más frecuente en la mujer posmenopáusica es el bochorno (85\%). Existen considerables variaciones en frecuencia, intensidad y duración entre las personas. Característicamente, es una sensación de calor que se inicia en la cara y cabeza, seguida de enrojecimiento facial que se irradia hacia el cuello y resto del cuerpo ${ }^{16}$ y puede durar de 1 a 5 minutos. Aunque su aparición coincide con la deprivación estrogénica, su etiología no puede explicarse totalmente por este hecho, ya que los niveles de estrógenos no difieren en la mujer asintomática y la sintomática menopáusica. ${ }^{17}$

El estudio PEPI fue una investigación multicéntrica de 3 años, aleatoria, doble enmascarada y controlada por placebo en 875 posmenopáusicas de 45 a 64 años. Su objetivo era examinar los efectos de los estrógenos solos o en combinación con 3 regímenes de progestágenos en determinadas consecuencias. Greendale y colaboradores confirman que los tratamientos hormonales orales mejoran los síntomas vasomotores comparados con placebo (OR $0.17-0.28$ ). No se encontró beneficio adicional de estrógenos conjugados asociados con acetato de medroxiprogesterona, sobre los estrógenos solos en el tratamiento de los bochornos. ${ }^{18}$

Nelson analiza todas las publicaciones desde 1966 hasta 2003, comparando la eficacia y la seguridad de las preparaciones estrogénicas más usadas para reducir los sofocos asociados con la menopausia. No se encontró ninguna diferencia significativa entre el estradiol transdérmico, el estradiol oral y los estrógenos conjugados solos o asociados con progesterona. Todos los 8 ensayos de estrógenos conjugados orales reportaron una mejoría en la frecuencia de los bochornos, su severidad, o ambos, al compararse con el placebo. No se documentó diferencia alguna entre las dosis de estrógenos conjugados y dosis bajas y continuas de acetato de medroxiprogesterona. ${ }^{19}$

La colaboración de Cochrane incluye a 2.511 pacientes de 21 estudios clínicos que cumplían los criterios de selección de ser aleatorios, a doble ciego y controlado con placebo, y al menos 3 meses de duración. Su conclusión fue que se redujo significativamente la frecuencia de sofocos y las sudoraciones nocturnas con terapia de reemplazo hormonal oral, en comparación con el placebo (OR de -17.46 con límites de confianza al 95\% de 124.72 a -10.21$).{ }^{20}$

\section{Prevención de eventos cardiovasculares}

Grady y colaboradores analizan 32 estudios epidemiológicos desde 1970, y encuentran evidencia de que el efecto protector de los estrógenos es mayor en las mujeres con enfermedad cardiaca previa. Mencionan que en una mujer de 50 años de edad, con el antecedente de enfermedad cardiaca, el uso de terapia hormonal incrementa en 2.1 años su expectativa de vida. ${ }^{12}$ Este hallazgo de disminución de la enfermedad cardiaca en las mujeres posmenopáusicas es un hecho consistente y biológicamente posible. ${ }^{21}$

El estudio PEPI demostró que las prescripciones usuales orales de estrógenos solos o asociados con el acetato de medroxiprogesterona, disminuyen el colesterol de baja densidad y aumentan el de alta densidad, pero no afectan la presión arterial, en comparación con el placebo. Todos son factores de riesgos de la enfermedad cardiaca en la mujer posmenopáusica. ${ }^{22}$ Sin embargo, resulta evidente que todas las formas de terapia de reemplazo hormonal oral incrementan los niveles de triglicéridos. ${ }^{23}$

Las investigaciones aleatorias brindaron nuevas directrices de tratamiento, pero también desencadenaron confusión en el ámbito mundial. ¿Qué pasó? ¿Pueden los resultados generalizarse a todos los tratamientos hormonales orales en las mujeres postmenopáusicas, o solo al tipo de medicamento usado en dichas investigaciones? 
El estudio HERS, con una duración inicial de 4.1 años, fue establecido para determinar el efecto de estrógenos conjugados y acetato de medroxiprogesterona, en forma oral y continua, al alterar el riesgo en mujeres posmenopáusicas con enfermedad coronaria establecida. Sus resultados muestran que no hubo ninguna diferencia entre el grupo de tratamiento y el control al reducir la tasa de enfermedad cardiovascular, a pesar de una disminución del 11\% en el nivel de las lipoproteínas de baja densidad y un aumento del $10 \%$ en las de alta densidad, en las pacientes con terapia hormonal. ${ }^{24}$

HERS II revisó los datos del estudio sobre el corazón y reemplazo de estrógenos y progesterona, que involucraba a 2763 mujeres con enfermedad cardiaca y edad promedio de 67 años. En contraste con el HERS inicial, aleatorio, enmascarado y controlado con placebo, el segundo fue abierto. Sus autores confirmaron los hallazgos de la primera investigación, en cuanto a que el mayor riesgo de enfermedad cardiaca coronaria ocurre en el primer año de tratamiento, y que no existe diferencia entre el grupo de tratamiento y el control sobre muertes de enfermedad coronaria o infartos de miocardio no fatales. ${ }^{25}$

El estudio WHI que valoraba los riesgos y beneficios de estrógenos y progesterona combinada en mujeres menopáusicas sanas, demostró un aumento significativo en enfermedad coronaria y riesgo de cáncer de seno invasivo, después de 5.2 años. Dicha investigación, asignada a terapia combinada hormonal oral versus placebo en forma aleatoria, señala beneficios al disminuir las fracturas de cadera y el cáncer de colon, pero el riesgo fue mayor que estos. ${ }^{26}$ Es preciso recordar que, en contraste con los estudios clínicos de tratamiento, los preventivos tienen reglas conservadoras para el cese de la investigación, pues los sujetos sanos no deben ser dañados por las estrategias preventivas de tratamiento ${ }^{27}$. Las conclusiones de tal análisis son: 1, el riesgo de $0.625 \mathrm{mg}$ de estrógenos conjugados más $2.5 \mathrm{mg}$ de acetato de medroxiprogesterona oral diarios, excede los beneficios luego de 5.2 años de seguimiento, en mujeres de 50 a 79 años; 2, esa dosis de estrógenos más progesterona no debe ser iniciada o continuada como prevención primaria de enfermedad cardiovascular coronaria; 3, el riesgo de enfermedad cardiovascular y cáncer de seno debe ser valorado contra los beneficios de fractura y cáncer de colon.

La parte del análisis WHI que evalúa el uso exclusivo de estrógenos para prevenir las enfermedades crónicas de las mujeres postmenopáusicas, muestra que no afecta la enfermedad coronaria en mujeres con histerectomía previa, luego de un seguimiento por 6.8 años. ${ }^{28}$ Las conclusiones sobre esta patología difieren del estudio HERS y WHI, uso de terapia hormonal combinada, en el sentido que estos mostraron un aumento en el riesgo durante el primer año de empleo. Tal discrepancia puede explicarse por el uso de progesterona, duración del estudio y seguimiento. ${ }^{29}$
Cochrane analiza todos los ensayos controlados aleatorios que comparan la terapia de reemplazo hormonal con placebo, por un mínimo de 6 meses. Su objetivo era evaluar los efectos de esta sobre la prevención primaria y secundaria de las enfermedades cardiovasculares en mujeres posmenopáusicas. No se encontró efecto protector sobre los resultados cardiovasculares evaluados..$^{30}$

Como consecuencia de las investigaciones aleatorias realizadas, se produjeron una serie de pronunciamientos de diferentes asociaciones médicas en contra del uso rutinario de estrógenos y progesterona para la prevención de condiciones crónicas en mujeres posmenopáusicas. ${ }^{31-34}$ Conviene tener en cuenta que los datos de tales estudios no pueden extrapolarse a mujeres sintomáticas menores de 50 años y solo aplican al grupo de mujeres para el que la investigación fue relevante. ${ }^{34}$

\section{Tratamiento de la atrofia vaginal y vulvar}

El hipoestrogenismo produce pérdida del contenido de colágeno y grasa de la vulva, disminución del espesor del epitelio vaginal y de sus trasudados, aumento de $\mathrm{pH}$ uretral y lesión de la función del esfínter uretral intrínseco. ${ }^{35}$ El síndrome clínico asociado con este deterioro de los tejidos urogenitales incluye irritación y sequedad vaginal, dispaurenia y frecuentes infecciones urinarias. ${ }^{36}$

El estudio WHI demuestra que el tratamiento continuo oral de estrógeno conjugado $\mathrm{y}$ el acetato de medroxiprogesterona mejoran la sequedad vaginal, en comparación con el placebo. ${ }^{37}$ Cardozo y colaboradores evidencian que el estrógeno en forma oral o vaginal y en cualquier dosis es efectivo en el tratamiento de la atrofia urogenital. La vía vaginal de administración se correlaciona mejor con el alivio de los síntomas manifestados por las pacientes. ${ }^{38}$ Probablemente, la terapia hormonal sistémica se indica en pacientes con síntomas menopáusicos asociados: bochornos y atrofia vaginal. ${ }^{36}$ Sin embargo, entre el $10 \%$ y el $25 \%$ de los pacientes con tratamiento de reemplazo oral persisten con síntomas de atrofia urogenital. ${ }^{38}$ La dosis y la duración del tratamiento es variable en cada caso. ${ }^{39}$

Las infecciones urinarias frecuentes son comunes en las mujeres postmenopáusicas. Cardozo refiere que la terapia con estrógenos orales o vaginales produce mejoría en el manejo de las infecciones urinarias recurrentes, aunque los tratamientos fueron variables en tiempo, tipos de estrógenos, vías de administración y dosis empleadas, en los estudios aleatorios. Sin embargo, los datos disponibles indican que la administración vaginal reduce la frecuencia de infecciones urinarias recurrentes en mujeres postmenopáusicas. ${ }^{40}$

Los tratamientos con estrógenos han sido recomendados para la incontinencia urinaria, patología frecuente en las mujeres postmenopáusicas. Grady y colaboradores reportan en 1525 pacientes del estudio HERS, que la dosis oral continua de estrógenos conjugados asociada con acetato de 
Terapia de reemplazo hormonal / Fernández-Morales D

medroxiprogesterona empeora la incontinencia urinaria, lo que se evidencia más a los 4 meses de tratamiento. ${ }^{41}$ Cochrane identifica 28 estudios que incluyen a 2926 mujeres con el diagnóstico sintomático o urodinámico de incontinencia urinaria de esfuerzo, de urgencia o mixto. Se concluye que el tratamiento con estrógenos, utilizando diferentes combinaciones de tipos, dosis, duración y seguimiento, puede mejorar la incontinencia de urgencia. La combinación de estrógenos y progesterona pareció reducir la mejoría. ${ }^{42}$

\section{Prevención de las osteoporosis posmenopáusica}

Los factores genéticos son responsables del 60-80\% de los determinantes de la masa ósea, la cual alcanza su máximo en la tercera década de la vida, el cual se mantiene hasta los 50 años, cuando existe una pérdida ósea relacionada con la edad. En las mujeres predomina una disminución ósea acelerada durante unos 5 ó 10 años después de la menopausia, que persiste con un ritmo menor durante el resto de la vida. ${ }^{43}$

Grady y colaboradores mencionan 11 estudios epidemiológicos hechos desde 1970 con solo estrógenos, que muestran un RR para fracturas de cadera de 0.75 (índice de confianza al 95\% de 0.68 a 0.84). ${ }^{12}$ Harlap refiere que es necesario un mínimo de 6 años de terapia de reemplazo estrogénica para reducir el riesgo de fractura de cadera y muñeca. ${ }^{1}$ Pero otros autores señalan que el estrógeno es más efectivo al prevenir fracturas en mujeres mayores de 75, que inician el tratamiento en un plazo de 5 años después de la menopausia y lo continúan indefinidamente. ${ }^{44}$

Aunque los estudios HERS no demostraron reducción en la incidencia de fractura ${ }^{45,46}$, la investigación WHI, con estrógenos conjugados y acetato de medroxiprogesterona orales en forma continua, refieren que esta combinación reduce el riesgo de fractura en la cadera, vértebras y muñeca. La OR para el riesgo de fractura de cadera fue de 0.67, con un índice de confianza al 95\%, de 0.47 a 0.96 ; para otras fracturas diferentes de columna, OR del $0.75 \%$ con índice de confianza al $95 \%$, de 0.68 a 0.83 . Dicho tratamiento produjo al sexto año un aumento consistente en la densidad de la masa ósea en la columna y la cadera, en comparación con el grupo placebo (7.5\% versus $2.6 \%) .{ }^{47} \mathrm{El}$ uso exclusivo de estrógenos también disminuye la incidencia total de fracturas en cadera y vértebras (OR de 0.70 con índice de confianza al $95 \%$ de 0.63 a 0.79$).{ }^{28}$

Desde el estudio PEPI que evalúa el efecto de la terapia hormonal a diferentes dosis de estrógenos asociados con progesterona versus placebo ${ }^{48}$, hasta los metaanálisis de los estudios aleatorios ${ }^{49,50}$, la terapia de reemplazo hormonal oral combinada tiene un efecto consistente y favorable en incrementar la densidad ósea en todos los sitios. Banks y colaboradores mencionan que todos los tipos de terapia hormonal confieren protección contra las fracturas, pero refieren que esta desaparece cuando cesa su uso. ${ }^{51}$
Greendale analiza los datos del trabajo PEPI y concluye que la pérdida ósea en las mujeres postmenopáusicas, mientras reciben terapia de reemplazo hormonal, es infrecuente para los diferentes sitios ${ }^{52}$, y que esta no es más acelerada que la que ocurre en mujeres que nunca recibieron tratamiento hormonal ${ }^{53}$. Greenpan y colaboradores, en un estudio a doble ciego y controlado con placebo, encuentran un incremento en la pérdida de masa ósea al suspender los estrógenos conjugados en la columna y la cadera. ${ }^{54}$ Todas estas consideraciones, asociadas a los riesgos reportados, han propiciado la recomendación de que la terapia de reemplazo hormonal no sea la primera elección al prevenir la osteoporosis. ${ }^{55}$

\section{Riesgos de la terapia hormonal oral}

En 1975 se encuentra una asociación entre el uso de estrógenos conjugados y el riesgo de cáncer de endometrio, la cual se incrementa con la duración de la exposición. ${ }^{2}$ El estudio PEPI demuestra un aumento en hiperplasia de endometrio, luego de tratamiento con solo estrógenos, que puede prevenirse con progesterona, en comparación con placebo. $^{5}$ Grady y colaboradores mencionan que se han publicado 35 estudios epidemiológicos desde 1970 hasta 1990, que indican que el riesgo de cáncer de endometrio se eleva con todas las dosis de estrógenos y aumenta según sean estas mayores. No se documenta mayor riesgo si las progestinas se usan al menos por 10 días cada mes. ${ }^{12}$

Grady revisa todos los estudios epidemiológicos que evalúan el efecto del tratamiento hormonal sobre el riesgo de cáncer de seno y enfermedad cerebrovascular. Aunque los resultados no son consistentes en ambas patologías, el RR es de 1.25 en tratamientos a largo plazo en el primero, $y$ de 0.96 en la segunda. ${ }^{12}$ El estudio HERS evidencia que existe un aumento de la enfermedad vesicular en las pacientes con terapia hormonal de reemplazo, y los eventos venosos tromboembólicos fueron 3 veces más comunes que en el grupo placebo, aunque la mortalidad total fue igual en ambos grupos $^{24}$, pero no hubo efecto significativo en el riesgo de enfermedad cerebrovascular de las postmenopáusicas con enfermedad coronaria. ${ }^{56}$ Idénticos riesgos fueron confirmados por el seguimiento de la investigación anterior de 6.8 años (HERS II). ${ }^{46}$

El estudio WHI refiere que el peligro de cáncer de seno estuvo directamente relacionado con la duración de la terapia. El incremento se manifiesta después de los 4 años de uso, principalmente en mujeres que antes han empleado terapia hormonal. El riesgo significativo de enfermedad coronaria y tromboembolismo venoso se pudo observar durante el primer año de tratamiento, aunque la enfermedad coronaria no se mantuvo elevada después. La tasa de mortalidad total no está afectada en enfermedad coronaria ni cáncer del seno en pacientes con terapia hormonal. ${ }^{26}$ El uso exclusivo de estrógenos como sustitución hormonal también aumenta el riesgo de enfermedad tromboembólica. ${ }^{28} \mathrm{La}$ misma investigación confirmó el mayor riesgo de accidentes 
cerebro vasculares en todos los subgrupos de las mujeres analizadas. ${ }^{57}$

El "Estudio del Millón de Mujeres”, una investigación de cohorte de 1084110 mujeres entre 50 y 64 años, fue establecido para indagar la relación entre el uso de terapia hormonal sustitutiva y la incidencia y mortalidad del cáncer de seno. Sus resultados confirmaron los hallazgos previos, relacionados con que usuarias actuales y recientes de terapia hormonal sustitutiva tienen un incremento en el riesgo de cáncer invasivo de mama, que se vincula con su tiempo de uso. El RR de terapia exclusiva con estrógenos es de 1.30, y el de estrógenos y progesterona de 2.00. La combinación de estrógenos conjugados y acetato de medroxiprogesterona, medicamentos usados en el estudio WHI, demostró un riesgo de 1.62 y 2.42, para una duración total de uso de menos de 5 años y más, respectivamente. Los autores sugieren que el estudio no muestra una gran diferencia entre el efecto de terapia con solo estrógenos y combinaciones de estrógenos y progesterona, ni el peligro de cáncer de mama difiere en los resultados entre los tipos específicos de estrógenos y progesterona. ${ }^{59}$

Anderson y colaboradores analizan a las mismas 16.608 mujeres del estudio WHI y buscan el efecto de la terapia hormonal continua y combinada en la incidencia de cáncer invasivo de ovario. La OR encontrada fue de 0.81 (intervalo de confianza al $95 \%$ de 0.77 a 3.24). ${ }^{58}$ Se comenta que la latencia del cáncer de ovario es de 25 años y es más probable que el tratamiento hormonal estimule tumores no diagnosticados en ese momento, en lugar de la transformación maligna per se. ${ }^{60}$

\section{Conclusiones}

El tratamiento para los síntomas vasomotores con estrógenos y progesterona debe restringirse a mujeres que no muestren factores de riesgo de enfermedad coronaria, por un periodo menor a 5.2 años. No se ha demostrado que esta contraindicación aplique para mujeres menopáusicas menores de 50 años. Ningún estudio ha sugerido que no se prescriba el estrógeno o estrógenos y progesterona, si la indicación es precisa. Solo se ha documentado que esa dosis y el tipo de terapia hormonal oral usada en los estudios mencionados, no debe ser iniciada o continuada como prevención primaria de condiciones crónicas en mujeres mayores de 50 años.

Los efectos reportados en los estudios aleatorios sobre enfermedad cardiovascular, tromboembolismo, cáncer de mama y accidentes cerebrovasculares son moderados en términos de riesgo absoluto, pero su implicación en la población que compromete a millones de usuarias es sustancial. Los resultados de estas investigaciones pueden expresar una subestimación del efecto real, por las limitaciones en cuanto a adherencia y entrecruzamiento de los grupos de tratamiento y control. Sus consecuencias se limitan al régimen de terapia de reemplazo hormonal oral utilizada y a pacientes mayores de 50 años.

El tratamiento de reemplazo hormonal de solo estrógenos o asociado con acetato de medroxiprogesterona es efectivo contra la atrofia urogenital. Sin embargo, la vía vaginal de administración se correlaciona mejor con el alivio de estos síntomas. El uso de terapia hormonal oral combinada, como indicación para prevenir la osteoporosis, no es la primera opción, sino que debe restringirse a pacientes que muestren contraindicaciones a otros medicamentos usados en tal patología y conozcan los riesgos.

Sigue siendo válido el criterio de que la evaluación individualizada del perfil de riesgo/beneficio de cada paciente, por un médico y una paciente informada, es el mejor curso de acción por seguir. El médico debe determinar la mínima dosis efectiva por el menor tiempo posible, con base en la preparación hormonal y su ruta de administración necesaria para alcanzar los objetivos terapéuticos que desea, en una situación clínica única.

\section{Abstract}

The use of oral hormonal replacement therapy has been controversial during the last years because randomized studies opposed all the previous guidelines. Some authors referred their observations and the limitations of these investigations. But it is demonstrated that the only indication that still persists is the treatment of the menopausal syndrome, using the lowest dose during the shortest time possible, and probably as a second option, in the management of osteoporosis. The objective of the present review to is support the medical physicians that prescribe hormonal replacement therapy for menopausal women older than 50 years of age.

\section{Referencias}

1. Harlap, S. The benefits and risks of hormone replacement therapy: An epidemiologic overview. Am J Obstet Gynecol 1992; 166:1986 1992

2. Ziel, H. Finkle, W. Increased risk of endometrial carcinoma among users of conjugated estrogens. N Engl J Med 1975; 293: 1167 - 1170

3. Udoff, L. Continuous estrogen-progestin replacement therapy in postmenopausal women. UpToDate, Rose, BD (Ed), UpToDate, Wellesley, MA, 2003. Recuperado el 10 de octubre del 2003 de http:// www.uptodate.com

4. Archer, D. Pickar, J. Bottiglioni, F. Bleeding patterns in postmenopausal women taking continuous combined or sequential regimens of conjugated estrogens with medroxiprogesterone acetate. Obstet Gynecol 1994;83: 686 - 692 
5. The Writing Group for the PEPI Trial. Effects of hormone replacement therapy on endometrial histology in postmenopausal women. JAMA 1996; 275: 370 - 375

6. Clisham, R. Ziegler, D. Lozano, K. Judd, H. comparison of continuous versus sequential estrogen and progestin therapy in postmenopausal Women. Obstet Gynecol 1991; 77: 241 - 246

7. Eiken, P. Kolthoff, N. Compliance with 10 years oral hormonal replacement therapy. Maturitas 2002; 41: 81 - 86

8. American College of Physicians. Guidelines for counseling postmenopausal women about preventive hormone therapy. Ann Intern Med 1992; 117:1038 - 1041

9. American College of Obstetricians and Gynecologists. Hormone replacement therapy. ACOG Technical Bulletin 166. Washington, DC: ACOG 1992

10. Nanas, H. Katz, D. American College of Preventive Medicine Practical policy statement, perimenopausal and postmenopausal hormone replacement therapy. Am J Prev Med 1999; 17: 250 - 254

11. Mosca, L. Grundy, S. Judelson, D. King, K. Limacher, M. Oparil, S. Guide to preventive cardiology for women. Circulation 1999;99: 2480 - 2484

12. Grady, D. Rubin, S. Petitti, D. Hormone Therapy to prevent disease and prolong life in postmenopausal women. Ann Intern Med 1992; 117: 1016 - 1037

13. Grimes D; Lobo R. Perspectives on the women's health initiative trial of hormone replacement therapy. Obstet Gynecol 2002; 100: 1344 1353

14. Keating. Cleary, P. Rossi, A. Zaslavsky, A. Ayanian, J. Use of hormone replacement therapy by postmenopausal women in the United States. Ann Intern Med 1999; 130: 545 - 553

15. Ravnikar, V. Compliance with hormone therapy. Am J Obstet Gynecol 1987; 156: 1332- 1334

16. Dawood, Y. Tidey, G. Menopause. Curr prob Obstet Gynecol Fertil 1993; 16: 169 - 208

17. Freedman, R. Pathophysiology and treatment of menopausal hot flashes. Semin Reprod Med 2005; 23: 117 - 125

18. Greendale, G. Reboussin, B. Hogan, P. Barnebei, V. Shumaker, S. Johnson, S. Barret-Connor, E. Symptom relief and side effects of postmenopausal hormones: results from the postmenopausal estrogen/ progestin intervention trial. Obstet Gynecol 1998; 92: 982 - 988.

19. Nelson, H. Commonly used types of postmenopausal estrogen for treatment of hot flashes. JAMA 2004; 291: 1610 - 1620

20. MacLennan, A. Lester, S. Moore, V. Terapia de reemplazo estrogénico por vía oral versus placebo para el tratamiento de los sofocos (Revisión Cochrane traducida) En: La Biblioteca Cochrane Plus, número 2, 2005. Oxford, Update Software Ltd. Recuperado el 5 de julio de 2005 de http://www.update-software.com

21. Barret-Connor, E. Estrogen and Estrogen - Progestogen replacement: therapy and cardiovascular diseases. Am J Med 1993; 95(Suppl 5A): $40 \mathrm{~S}-43 \mathrm{~S}$

22. The Writing Group for the PEPI Trial. Effects of estrogen or estrogen/ progestin regimens on heart disease risk factor in postmenopausal women: the Postmenopausal Estrogen / Progestin Interventions (PEPI) Trial. JAMA 1995; 273: 199 - 208

23. Editorials. PEPI in Perspective. Good answers spawn pressing Questions. JAMA 1995; 273: 240 - 241.

24. Hulley S; Grady D; Bus T; Furberg C; Herrington D; Riggs BL; Vittinghoff E. Randomized trial el estrogen plus progestin for secondary prevention of coronary heart disease in postmenopausal women. Heart and Estrogen / progestin Replacement Study (HERS) Research Group. JAMA 1998; 280: 605 - 613

25. Grady D; Herrington D; Bittner V; Blumenthal R; Davidson M; Hlatky M; Hsia J; Hulley S; Herd A; Khan S; Newby LK; Waters D; Vitinghoff $\mathrm{E}$; Wenger N. Cardiovascular disease outcomes during 6.8 years of hormone therapy: Heart and Estrogen/progestin Replacement Study follow - up (HERS II). JAMA 2002; 288: 49 - 57
26. Writing Group for the Women's Health Initiative Investigators. Risk and benefits of estrogen plus progestin in health postmenopausal women: principal results from the women's health initiative randomized controlled trial. JAMA 2002; 288:321- 333

27. Liu, J. Menopausal hormone therapy: a paradigm shift? Fertil Steril 2003; 80: 494 - 495

28. The Women's Health Initiative Steering Committee. Effects of conjugated equine estrogens in postmenopausal women with hysterectomy. The womens health initiative randomizad controlled Trial. JAMA 2004; 291: 1701 - 1712

29. Hulley, S. Grady, D. The WHI Estrogen-Alone Trial - Do Things Look Any Better? JAMA 2004, 291: 1769 - 1771

30. Sánchez, G. Carmona, L. Roque, M. Sánchez, L. Bonfill, X. Terapia de reemplazo hormonal para la prevención de enfermedades cardiovasculares en mujeres posmenopáusicas (Revisión Cochrane traducida) En: La Biblioteca Cochrane Plus, 2005. Oxford: Update Software Ltd. Recuperado el 7 de agosto de 2005 de http://www. update-software.com

31. U.S. Preventive Services Task Force. Postmenopausal hormone replacement therapy for primary prevention of chronic conditions: recommendations and rationales. Ann Intern Med 2002; 137:834 839

32. Sociedad Norteamericana de Menopausia. Informe del panel asesor de la NAMS sobre la terapia hormonal de la posmenopáusica. Menopause 2003; 10: 6 - 12

33. Recommendation statement from the Canadian task force on preventive health care. Postmenopausal hormone replacement therapy for primary prevention of cardiovascular and cerebrovascular disease. CMAJ 2004; 170: 1388 - 1390

34. North American Menopause Society. Recommendations for estrogen and progesterone use in peri and postmenopausal women: October 2002 position statement of the North American Menopause Society. Menopause 2004; 11:589 - 600

35. Ballagh, S. vaginal hormone therapy for urogenital and menopausal symptoms. Sem Reprod Med 2005; 23: 126 - 140

36. SOGC Clinical Practice Guidelines. The detection and management of vaginal atrophy. J Obstet Gynecol Can 2004; 26: 503 - 508

37. Barnabei, V. Cochrane, B. Aragaki, A. Nygaard, I. Williams, S. McGovern, P. Menopausal symptoms and treatment-related effects of estrogen and progestin in the women's health initiative. Obstet Gynecol 2005; 105: 1063 - 1073

38. Cardozo. L. Bachmann, G. McClish, D. Fonda, D. Birgerson, L. MetaAnalysis of estrogen therapy in the management of urogenital atrophy in postmenopausal women: Second Report of the Hormones and Urogenital Therapy Committee. Obstet Gynecol 1998; 92: 722 - 727

39. Bachmann, G. Nevadunsky, N. Diagnosis and treatment of atrophic vaginitis. Am Fam Physician 2000; 61: 3090 - 3096

40. Cardozo, L. Lose, G. McClish, D. Versi, E. Koning, H. A Systematic review of estrogens for recurrent urinary tract infections: Third report of the hormones and urogenital therapy committee. Int Urogynecol J 2001; $12: 15-20$

41. Grady, D. Brown, J. Vittinghoff, E. Applegate, W. Varner, E. Snyder, T. Postmenopausal hormones and incontinence: The heart and estrogen/ progestin replacement study. Obstet Gynecol 2001; 97: 116 $-120$

42. Moehrer, B. Hextall, A. Jackson, S. Estrógenos para la incontinencia urinaria en mujeres (Revisión Cochrane traducida) En: La Biblioteca Cochrane Plus, 2005 Número 3, Oxford: Update Software Ltd. Recuperado el 2 de febrero de 2006 de http://www.update-software. com

43. Compton, J. Sex steroids and bone. Physiological Reviews 2001; 81: $420-437$

44. Couley, J. Seeley, D. Ensrud, K. Ettinger, B. Black, D. Cummings, S. Estrogen replacement therapy and fractures in older women. Ann Intern Med 1995; 122: 9 - 12 
45. Cauley, J. Black, D. Barrett-Connor, E. Harris, F. Shields, K. Applegate, W. Cummings, R. Effects of hormones replacement therapy on clinical fractures and height loss: the heart and estrogen/ progestin replacement study (HERS). Am J Med 2001; 110: 442 450

46. Hulley, S. Furberg, C. Barrett-Connor, E. Cauley, J. Grady, D. Haskell, W. Noncardiovascular Disease Outcomes During 6.8 Years of hormone therapy. Heart and estrogen/progestin replacement study follow-up (HERS II). JAMA 2002; 288: 58 - 64

47. Cauley, J. Robbins, J. Chen, Z. Cummings, S. Jackson, R. LaCroix, A. Effects of estrogen plus progestin on risk of fracture and bone mineral density. The women's health initiative randomized trial. JAMA 2003; 290: 1729 - 1738

48. The Writing Group for the PEPI. Effects of hormone therapy on bone mineral density: results from the postmenopausal estrogen/progestin interventions ( PEPI) trial. JAMA 1996; 276: 1389 - 1396

49. Torgerson, D. Bell-Syer, S. Hormone replacement therapy and prevention of nonvertebral fractures. A meta-analysis of randomized trial. JAMA 2001; 285: 2891 - 2897

50. Wells, G. Tugwell, P. Shea, B. Guyatt, G. Peterson, J. Zytaruk, N. Meta-Analysis of the efficacy of hormone replacement therapy in treating and preventing osteoporosis in postmenopausal women. Endoc Reviews 2002; 23: 529 - 539

51. Banks, E. Beral, V. Reeves, G. Balkwill, A. Barnes, I. Fracture incidence in relation to the pattern of use of hormone therapy in postmenopausal women. JAMA 2004; 291: 2212 - 2220

52. Greendale, G. Wells, B. Marcus, R. Barrett-Connor, E. How many women lose bone mineral density while taking hormone replacement therapy? Results from the postmenopausal estrogen/progestin interventions trial. Arch Intern Med 2000; 160: 3065 - 3071
53. Greendale, G. Espeland, M. Stone, S. Marcus, R. Barrett-Connor, E. Bone mass response to discontinuation of long-term hormone replacement therapy. Results from the postmenopausal estrogen/ progestin interventions (PEPI) safety follow-up study. Arch Intern Med 2002; 162: 665 - 672

54. Greenspan, S. Emkey, R. Bone, H. Weiss, S. Bell, N. Downs, R. Significant differential effects of alendronate, estrogen or combination therapy of the rate of bone loss after discontinuation of treatment of postmenopausal osteoporosis. Ann Intern Med 2002; 137: 875 - 883

55. Cheung, A. Feig, D. Kapral, M. Diàz, N. Dodin, S. and the Canadian task force on preventive health care. CMAJ 2004; 170: 1665 - 1667

56. Simon, J. Hsia, J. Cauley, J. Richard, C. Harris, F. Fong, J. Postmenopausal hormone therapy and risk of stroke. The heart and estrogen-progestin replacement study. Circulation 2001;103: 638 642

57. Wassertheil, S. Hendrix, S. Limacher, M. Heiss, G. Kooperberg, C.Baird, A. Effect of estrogen plus progestin on stroke in postmenopausal women. The women's health initiative: a randomized trial. JAMA 2003, 289: 2673 - 2684

58. Million Women Study Collaborators. Breast cancer and hormonesreplacement therapy in the million women study. Lancet 2003; 362: $419-427$

59. Anderson, G. Judd, H. Kaunitz, A. Barad, D. Beresford, S. Pettinger, M. Effects pf estrogen plus progestin on gynecologic cancers and associated diagnostic procedures. The women's health initiative randomized trial. JAMA 2003; 290: 1739 - 1748

60. Risch, H. Postmenopausal estrogen - only, but not estrogen + progestin, was associated with increased risk of ovarian cancer. Evid based Obstet Gynecol 2003; 5: 53 - 54 